\title{
REGULARITY OF A PARABOLIC FREE BOUNDARY PROBLEM WITH HÖLDER CONTINUOUS COEFFICIENTS
}

\author{
ANDERS EDQUIST AND ERIK LINDGREN
}

ABstract. We consider the parabolic obstacle type problem

$$
\left\{\begin{array}{l}
H u=f \chi_{\Omega} \text { in } Q_{1}^{-}, \\
u=|\nabla u|=0 \text { on } Q_{1}^{-} \backslash \Omega,
\end{array}\right.
$$

where $\Omega$ is an unknown open subset of $Q_{1}^{-}$. This problem has its origin in parabolic potential theory. When $f$ is Hölder continuous we can, under a combination of energetic and geometric assumptions, prove the optimal $C_{x}^{1,1} \cap$ $C_{t}^{0,1}$ regularity of the solution.

\section{INTRODUCTION AND MAIN RESULT}

1.1. Problem. Let $u$ solve

$$
\left\{\begin{array}{lll}
H u=f \chi_{\Omega} & \text { in } & Q_{1}^{-}, \\
u=|\nabla u|=0 & \text { in } & Q_{1}^{-} \backslash \Omega .
\end{array}\right.
$$

Here $Q_{R}^{-}=B_{R} \times\left(-R^{2}, 0\right], H$ is the heat operator, $\Lambda=\{u=|\nabla u|=0\}$ and $\Omega=Q_{1}^{-} \backslash \Lambda$. For the function $f$ we assume Hölder continuity in both space and time.

This problem arises in parabolic potential theory as follows. Let $g(x, t)$ be a function with support in $\Omega \subset Q_{1}^{-}$, and

$$
G(x, s)=\frac{1}{(4 \pi s)^{n / 2}} e^{\frac{-|x|^{2}}{4 s}}
$$

be the heat kernel.

The heat potential can be defined as

$$
U[g](x, t)=g(x, t) * G(x,-t),
$$

where the convolution is taken over $\mathbb{R}^{n} \times \mathbb{R}^{+}$.

Since $G$ is the heat kernel we have that

$$
H U[g](x, t)=c_{n} g(x, t)=f \chi_{\Omega} \text { in } \mathbb{R}_{n} \times \mathbb{R}^{+},
$$

where $c_{n}$ is a negative constant depending only on the dimension and $f$ is a nonzero function. If there is a function $v$ that fulfills

$$
\begin{array}{lll}
H v=0 & \text { in } & Q_{1}^{-}, \\
v=U[g] & \text { in } & Q_{1}^{-} \backslash \Omega,
\end{array}
$$

Date: June 17, 2010.

This research project is a part of the ESF program Global. Moreover, E. Lindgren has partially been supported by grant KAW 2005.0098 from the Knut and Alice Wallenberg foundation, the ANR project "MICA", grant ANR-08-BLAN-0082 and by the Chair "Mathematical modelling and numerical simulation, F-EADS - Ecole Polytechnique - INRIA" during this work. 
then we refer to $v$ as the caloric continuation of $U[g]$. When the boundary of a domain is analytic the existence of such a caloric continuation is locally guaranteed by Cauchy-Kowalevskaya theorem.

Let $u=U[g]-v$, then $u$ will satisfy

$$
\begin{array}{lll}
H u=f \chi_{\Omega} & \text { in } & Q_{1}^{-} \\
u=|\nabla u|=0 & \text { in } & Q_{1}^{-} \backslash \Omega,
\end{array} .
$$

which explains why we study (1.1).

1.2. Notation. Throughout the paper we will use the following notation:

$B_{r}\left(x_{0}\right)=\left\{x \in \mathbb{R}^{n}:\left|x-x_{0}\right|^{2}<r\right\}$ the open spatial ball

$Q_{r}^{-}\left(x_{0}, t_{0}\right)=B_{r}\left(x_{0}\right) \times\left(-r^{2}+t_{0}, 0+t_{0}\right]$ the lower half cylinder

$S_{r}=\mathbb{R}^{n} \times\left(-4 r^{2}, 0\right]$ an infinite strip

$T_{r}=\mathbb{R}^{n} \times\left(-4 r^{2},-r^{2}\right]$ another infinite strip

$G(x, s)=\frac{1}{(4 \pi s)^{n / 2}} e^{\frac{-|x|^{2}}{4 s}}$ the heat kernel

$\mathrm{d} \gamma(x, s)=G(x,-s) \mathrm{d} x \mathrm{~d} t$

$\mathrm{d} \gamma^{s}(x)=G(x,-s) \mathrm{d} x$

$\|f\|_{L_{\mu}^{p}(D)}=\left(\int_{D}|f(y)|^{p} \mathrm{~d} \mu\right)^{1 / p}$ Lebesgue norm for a measure $\mu$

$\|f\|_{H_{\mu}(D)}=\left(\int_{D}|f(y)|^{2}+|\nabla f(y)|^{2} \mathrm{~d} \mu\right)^{1 / 2}$ Sobolev norm for a measure $\mu$

$\|f\|_{N\left(D_{1} ; M\left(D_{2}\right)\right)}=\|g(t)\|_{M\left(D_{2}\right)}$ where $g(t)=\|f(\cdot, t)\|_{N\left(D_{1}\right)}$

$\|f\|_{W_{p}^{2,1}\left(Q_{r}^{-}\right)}=\|f\|_{L^{p}\left(Q_{r}^{-}\right)}+\|\nabla f\|_{L^{p}\left(Q_{r}^{-}\right)}+\left\|D^{2} f\right\|_{L^{p}\left(Q_{r}^{-}\right)}+\left\|f_{t}\right\|_{L^{p}\left(Q_{r}^{-}\right)}$

$u_{r}(x, t)=\frac{u\left(r x, r^{2} t\right)}{r^{2}}$ the parabolic rescaling

1.3. Known results. In [CPS04] the same parabolic problem was studied but with the restriction $f=1$. For this case the local $C_{x}^{1,1} \cap C_{t}^{0,1}$ regularity of the solution and the analyticity of the free boundary under some geometric assumption was proved.

The corresponding elliptic problem has been studied earlier. In [CKS00] the $C^{1,1}$ regularity of the solution was proved for constant $f$. Under a geometric condition on $\Omega$ near the origin it was also proved that the free boundary is analytic in a neighborhood of the origin.

A more general elliptic problem was studied in [PS07a]. The authors made the following double Dini assumption on the modulus of continuity of $f$ :

$$
\int_{0^{+}} \frac{\omega(r) \log \frac{1}{r}}{r} \mathrm{~d} r<\infty
$$

Under a combination of energetic and geometric assumptions they were able to prove $C^{1,1}$ regularity of the solution and $C^{1}$ regularity of the free boundary. In our case, due to certain technical issues for the heat equation we have to make the condition on $f$ slightly stronger.

The parabolic obstacle problem, i.e. the case $u \geq 0$, which has an important application in valuation of stock options of American type, has been studied before. In [PS07b], it was proved that the local regularity of the solution is $C^{1,1} \cap C^{0,1}$. The option application was also studied in [LS09]. Under the assumption that the pay-off of an American option on serveral assets is convex, it was proved that the free boundary is the graph of a $C^{\infty}$ function.

1.4. Main result. The main result in this paper is that solutions of (1.1) are, under geometric and energetic assumptions, $C_{x}^{1,1} \cap C_{t}^{0,1}$ regular if $f$ is Hölder continuous. The regularity of the free boundary is left as an open problem, the reason being 
that there is a bunch of results that needs to be proved on the way to the regularity of the free boundary, for instance the results in [Bla01] and [BS03]. This could be an interesting future project.

In order to state our main theorem more precisely we define the class of solution we consider in this paper.

Definition 1. We say $u$ is a local solution in $P_{R}\left(M_{1}, M_{2}\right)$ if

(1) $H u=f \chi_{\Omega}$, in $Q_{R}^{-}$, in the sense of distributions,

(2) $u=|\nabla u|=0$ in $Q_{R}^{-} \backslash \Omega$,

(3) $\sup _{Q_{R}^{-}}|u| \leq M_{1}$,

(4) for some $0<\beta<1$ there holds

$$
\sup _{\left|x-x_{0}\right| \leq r,\left|t-t_{0}\right| \leq r^{2}}\left|f(x, t)-f\left(x_{0}, t_{0}\right)\right| \leq M_{2} r^{\beta},
$$

for all $r$ small enough.

(5) $f(0,0)=1$,

(6) $(0,0) \in \Gamma=\partial \Omega \cap Q_{R}^{-}$.

We also need the following definition:

Definition 2. The parabolic thickness function is given by

$$
\delta(r, u)=\frac{\min \operatorname{diam}\left(\Lambda\left(u\left(\cdot,-r^{2}\right)\right) \cap B_{r}\right)}{r} .
$$

Our main theorem is the following:

Theorem 1. Assume $u \in P_{4}\left(M_{1}, M_{2}\right), \psi \in C_{0}^{\infty}\left(B_{1}\right), \psi=1$ in $B_{\frac{1}{2}}$ and $v=u \psi$. Then for given $\varepsilon>0$ there exists $r_{\varepsilon, M_{1}, M_{2}}>0$ such that if for some $0<r_{0}<$ $r_{\varepsilon, M_{1}, M_{2}}$

$$
\delta\left(r_{0} / 2, v\right) \geq \varepsilon \text { and } W\left(r_{0} ; v, f\right)<2 A_{n}-\varepsilon,
$$

then

$$
\|v\|_{L^{\infty}\left(Q_{r}^{-}\right)} \leq C r^{2} \text { for every } 0<r \leq r_{0}
$$

and

$$
\|v\|_{C_{x}^{1,1} \cap C_{t}^{0,1}\left(Q_{c_{0}}^{-}\right)} \leq C
$$

for some small $c_{0}=c\left(\varepsilon, M_{1}, M_{2}, r_{0}\right)$ and $C$ a fixed constant. Here $A_{n}=\frac{15}{4}$ is related to the energy of global solutions, see (Theorem I in [CPS04]).

\section{WEISS' MONOTONICITY FORMULA}

Define the Weiss energy for $v(x, t): \mathbb{R}^{n} \times \mathbb{R}^{-} \rightarrow \mathbb{R}$ to be

$$
W(r ; v, f)=\frac{1}{r^{4}} \int_{T_{r}}\left(|\nabla v|^{2}+2 f v+\frac{v^{2}}{t}\right) \mathrm{d} \gamma,
$$

and let

$$
v_{r}(x, t)=\frac{v\left(r x, r^{2} t\right)}{r^{2}},
$$

so that $W(r ; v, f)=W\left(1 ; v_{r}, f_{r}\right)$, where $f_{r}(x, t)=f\left(r x, r^{2} t\right)$.

The following proposition is a parabolic version of Weiss' monotonicity formula. 
Proposition 1. Let $u \in P_{1}\left(M_{1}, M_{2}\right), \psi \in C_{0}^{\infty}\left(B_{3 / 4}\right)$ such that $\psi=1$ on $B_{1 / 2}$, and set $v=u \psi$. Then there is a $W^{1,1}$-function $F=F_{M_{1}, M_{2}}$ such that

$$
W(r ; v, f)+\int_{0}^{r} F(s) \mathrm{d} s
$$

is a nondecreasing function for $0<r<1 / 2$, and in particular

$$
W^{\prime}(r ; v, f) \geq-F(r)+\frac{1}{r^{4}} \int_{T_{r}} \frac{(L v)^{2}}{-t} \mathrm{~d} \gamma .
$$

We make the following definition of a parabolic homogeneous function.

Definition 3. We say that $h$ is parabolic homogeneous of degree $\alpha$ if $h\left(k x, k^{2} t\right)=$ $k^{\alpha} h(x, t)$.

Using this definition we state a remark for the Weiss energy for $M_{2}=0$.

Remark 1. For $M_{2}=0$ we have $f=1$. Let $u \in P_{\infty}\left(M_{1}, 0\right)$ then $W(r ; u, 1)$ is nondecreasing in $r$. Furthermore $W(r ; u, 1)$ is constant for $r_{0}<r<r_{1}$ if and only if $u$ is parabolic homogeneous of degree 2 in $Q_{r_{1}}^{-} \backslash Q_{r_{0}}^{-}$. This was proved in [Wei99].

In order to prove the proposition we first establish two technical results. The first result is an estimate for a linear operator applied to a local solution.

Lemma 1. Let $u \in P_{1}\left(M_{1}, M_{2}\right)$ and define

$$
L u=x \cdot \nabla u+2 t u_{t}-2 u .
$$

Then for any $\alpha \in(0,1)$ there is a constant $C_{\alpha}$ such that

whenever $r<\frac{1}{2}$.

$$
\frac{1}{\left|Q_{r}^{-}\right|} \int_{Q_{r}^{-}} \frac{L u}{r^{2}} \mathrm{~d} x \mathrm{~d} t \leq \frac{C_{\alpha}}{r^{\alpha}},
$$

Proof. As a special case of Theorem 4.8 in [Lie96] we find that $u \in C_{x}^{1,1-\alpha} \cap C_{t}^{0,1-\alpha}$ for any $\alpha \in(0,1)$ with the estimates depending on $\alpha, M_{1}$ and $M_{2}$.

Define

Then, by the regularity of $u$

$$
v_{r}(x, t)=\frac{u\left(r x, r^{2} t\right)}{r^{2}} .
$$

$$
\sup _{Q_{1}^{-}}\left|v_{r}(x, t)\right| \leq \frac{C}{r^{\alpha}}
$$

when $r<\frac{1}{2}$. Moreover,

$$
\sup _{Q_{1}^{-}}\left|H v_{r}\right| \leq C .
$$

Therefore, by $L^{p}$-estimates (Theorem 7.22 in [Lie96])

$$
\left\|v_{r}\right\|_{W_{p}^{2,1}\left(Q_{1}^{-}\right)} \leq \frac{C}{r^{\alpha}} .
$$

In particular for $p=1$ we have

$$
\left\|\nabla v_{r}\right\|_{L^{1}\left(Q_{1}^{-}\right)}+\left\|v_{r}\right\|_{L^{1}\left(Q_{1}^{-}\right)}+\left\|\partial_{t} v_{r}\right\|_{L^{1}\left(Q_{1}^{-}\right)} \leq \frac{C}{r^{\alpha}} .
$$

Scaling back, this gives the desired estimate for $L u$.

The next technical result gives integral estimate over $B_{1} \times\left(-4 r^{2},-r^{2}\right)$ for an integrable function. 
Lemma 2. Suppose $k \in C^{\beta}\left(Q_{1}^{-}\right)$with $k(0,0)=0$, and $g \in L^{1}\left(Q_{1}^{-}\right)$with

$$
\left(\frac{1}{\left|Q_{r}^{-}\right|} \int_{Q_{r}^{-}}\left|\frac{g}{r^{2}}\right| \mathrm{d} x \mathrm{~d} t\right) \leq \frac{C_{\alpha}}{r^{\alpha}}
$$

Then

$$
\frac{1}{r^{4}} \int_{-4 r^{2}}^{-r^{2}} \int_{B_{1}}|k g| \mathrm{d} \gamma \leq C_{0} r^{\beta-\alpha}
$$

where $C_{0}=C(\alpha, \beta, n)$.

Proof. The proof is split into two parts, estimates inside and outside $B_{r}$. We start with the part that comes from integration only over $B_{r}$. For $(x, s) \in T_{r}$ we have

$$
|G(x, s)| \leq \frac{C}{r^{n}} .
$$

Therefore, we obtain

$$
\frac{1}{r^{4}} \int_{-4 r^{2}}^{-r^{2}} \int_{B_{r}}|k g| \mathrm{d} \gamma \leq C \frac{1}{r^{n+2}} \int_{-4 r^{2}}^{0} \int_{B_{r}} C_{\beta} r^{\beta} \frac{|g|}{r^{2}} \mathrm{~d} x \mathrm{~d} t \leq C_{0} r^{\beta-\alpha} .
$$

Now we turn our attention to what happens outside $B_{r}$. We split $B_{1} \backslash B_{r}$ into dyadic rings of the form $B_{2^{k+1} r} \backslash B_{2^{k} r}$. We observe that for $(x, s) \in B_{2^{k+1} r} \backslash B_{2^{k} r} \times$ $\left(-4 r^{2},-r^{2}\right)=C_{r}$ we have for some $c>0$,

Therefore,

$$
c \frac{e^{-\frac{1}{c} 4^{k}}}{r^{n}} \leq G(x, s) \leq \frac{e^{-c 4^{k}}}{c r^{n}} .
$$

$$
\begin{array}{r}
\frac{1}{r^{4}} \int_{C_{r}}|k g| \mathrm{d} \gamma \leq \frac{e^{-c 4^{k}}}{c r^{n+4}} \int_{C_{r}}|k g| \mathrm{d} x \mathrm{~d} t \\
\leq \frac{e^{-c 4^{k}}}{c r^{n+2}}\left(2^{k+1}\right)^{2} \int_{Q_{2^{k+1} r}^{-}} C_{\beta} r^{\beta} \frac{|g|}{\left(2^{k+1} r\right)^{2}} \mathrm{~d} x \mathrm{~d} t \\
\leq C e^{-c 4^{k}} r^{\beta-\alpha} 2^{(k+1)(n+2)} .
\end{array}
$$

Adding up all the rings we obtain

$$
\begin{aligned}
& \frac{1}{r^{4}} \int_{B_{1} \backslash B_{r}}|k g| \mathrm{d} \gamma^{s} \leq \sum_{k=1}^{2^{k+1} r=1} \frac{1}{r^{4}} \int_{C_{r}}|k g| \mathrm{d} \gamma \\
& \leq r^{\beta-\alpha} \sum_{1}^{\infty} C e^{-c 4^{k}} 2^{(k+1)(n+2)} \leq C r^{\beta-\alpha} .
\end{aligned}
$$

Combining (2.1) and (2.2) gives the desired estimate.

We use Lemma 1 and 2 to prove of Proposition 1.

Proof of Proposition 1. Let

$$
E(r ; v)=\frac{1}{r^{4}} \int_{T_{r}}\left(|\nabla v|^{2}+2 v+\frac{v^{2}}{t}\right) \mathrm{d} \gamma .
$$

We observe that

$$
E(r s ; v)=E\left(s ; v_{r}\right)
$$

with

$$
v_{r}(x, t)=\frac{v\left(r x, r^{2} t\right)}{r^{2}}
$$


Moreover, it is clear that

$$
W(r ; v, f)-E(r ; v)=\frac{1}{r^{4}} \int_{T_{r}} 2(f-1) v \mathrm{~d} \gamma .
$$

Define

$$
L v=\left.\frac{\mathrm{d}}{\mathrm{d} r} v_{r}\right|_{r=1}=x \cdot \nabla v+2 t v_{t}-2 v .
$$

Using that $\nabla G(x,-t)=\frac{x}{2 t} G(x,-t)$ and $\nabla v \nabla L v=\nabla \cdot(\nabla v L v)-\Delta v L v$, integration by parts gives

$$
\begin{aligned}
E^{\prime}(r, v) r & =\left.\frac{\mathrm{d}}{\mathrm{d} p} E(p r ; v)\right|_{p=1} \\
& =\frac{1}{r^{4}} \int_{T_{r}}\left(2 \nabla v \nabla L v+2 L v+\frac{2 v L v}{t}\right) G(x,-t) \mathrm{d} x \mathrm{~d} t \\
& =\frac{1}{r^{4}} \int_{T_{r}}\left(2 \nabla \cdot \nabla v L v-2 \Delta v L v+2 L v+\frac{2 v L v}{t}\right) G(x,-t) \mathrm{d} x \mathrm{~d} t \\
& =\frac{1}{r^{4}} \int_{T_{r}} L v\left(-\frac{x}{t} \cdot \nabla v-2 H v-2 v_{t}+2 L v+\frac{2 v}{t}\right) G(x,-t) \mathrm{d} x \mathrm{~d} t \\
& =\frac{1}{r^{4}} \int_{T_{r}} L v\left(2-2 H v+\frac{2 v-2 t v_{t}-x \cdot \nabla v}{t}\right) G(x,-t) \mathrm{d} x \mathrm{~d} t \\
& =\frac{1}{r^{4}} \int_{T_{r}} L v\left(2(1-H v)-\frac{L v}{t}\right) G(x,-t) \mathrm{d} x \mathrm{~d} t \\
& =\frac{2}{r^{4}} \int_{T_{r}} L v(1-H v) \mathrm{d} \gamma+\frac{1}{r^{4}} \int_{T_{r}} \frac{(L v)^{2}}{-t} \mathrm{~d} \gamma
\end{aligned}
$$

These calculations can be justified by taking a smooth approximation of $v$. We have to prove that the first term is integrable. We split it into two pieces:

$$
\begin{array}{r}
\frac{1}{r^{4}} \int_{T_{r}} L v(f(0)-f) \mathrm{d} \gamma \\
+\frac{1}{r^{4}} \int_{T_{r}} L v(f-H v) \mathrm{d} \gamma=A+B
\end{array}
$$

We observe that $L v$ vanishes outside $B_{1}$. Therefore, by Lemma 1 combined with Lemma 2

$$
\int_{0}^{1} A \leq C \int_{0}^{1} r^{\beta-\alpha} \leq C
$$

if $\alpha<\beta$. The term $B$ can be estimated quite easily since $f-H v=0$ in $B_{1 / 2}$. Hence, we have only contributions from $B_{\frac{3}{4}} \backslash B_{\frac{1}{2}}$, where $G(x,-s) \leq C r^{-n} e^{-\frac{1}{64 r^{2}}}$ and clearly $(f-H v) \in L^{\infty}\left(Q_{1}^{-}\right)$. Therefore

$$
\begin{aligned}
& |B|=\frac{1}{r^{4}} \int_{-4 r^{2}}^{-r^{2}} \int_{B_{\frac{3}{4}} \backslash B_{\frac{1}{2}}}|L v(f-H v)| \mathrm{d} \gamma \\
& \leq C \frac{e^{-\frac{1}{64 r^{2}}}}{r^{n+4}} \int_{Q_{1}^{-}}|L v| \mathrm{d} x \mathrm{~d} t \leq \frac{C}{r^{n+4}} e^{-\frac{1}{64 r^{2}}},
\end{aligned}
$$

which is integrable. It remains to prove that $W(r ; v, f)-E(r ; v) \rightarrow 0$ as $r \rightarrow 0$. Since $v$ has support only in $B_{1}, v \in C^{1, \alpha}$ and $f \in C^{\beta}$ we can apply Lemma 2 with $\alpha=0$, and obtain

$$
|W(r ; v, f)-E(r ; v)|=\left|\frac{1}{r^{4}} \int_{T_{r}} 2(f-1) v \mathrm{~d} \gamma\right| \leq C r \rightarrow 0 .
$$




\section{TECHNICAL TOOLS}

In this section we will prove and state some technical results needed for the proofs later. The first one, given below, is a result due to Simon (see Theorem 6 p. 86 in [Sim87]).

Lemma 3. (Simon's lemma) Let $X_{0} \subset X \subset X_{1}$ be Banach spaces such that $X_{0}$ is compactly embedded in $X$ and $X$ is continuously embedded in $X_{1}$. Moreover assume that $u_{k}$ is a sequence of functions such that for some $q>1$

$$
\left\|u_{k}\right\|_{L^{q}(I ; X)}+\left\|u_{k}\right\|_{L^{1}\left(I ; X_{0}\right)}+\left\|\partial_{t} u_{k}\right\|_{L^{1}\left(I ; X_{1}\right)} \leq C
$$

where $I \subset \mathbb{R}$ is a compact interval, then there is a subsequence $u_{k_{j}}$ that converges in $L^{p}\left(I ; X_{0}\right)$ for all $1 \leq p<q$.

In what follows we will apply this lemma with $X_{0}=H_{\gamma^{-1}}^{1}\left(\mathbb{R}^{n}\right), X=L_{\gamma^{-1}}^{2}\left(\mathbb{R}^{n}\right)$ and $X_{1}=(Y)^{*}$, the dual of the space $Y$ equipped with the norm

$$
\|\phi\|_{Y}=\left\|\frac{\phi}{G(x, 1)}\right\|_{L^{\infty}\left(\mathbb{R}^{n}\right)}+\|\nabla \phi\|_{L_{\left(\gamma^{-1}\right)^{-1}}^{2}},
$$

where the subscript $\gamma^{-1}$ or $\left(\gamma^{-1}\right)^{-1}$ stands for that we use the weighted space with $\gamma^{-1}=G(x, 1)$ or $\left(\gamma^{-1}\right)^{-1}$ as weight. Then, the fact that the embedding $X \rightarrow X_{0}$ is compact is a special case of Theorem 3.1 in [Hoo81]. The fact that the embedding $X \rightarrow X_{1}$ is continuous is clear. We will also use that we have an embedding of the form

$$
\nabla L_{\gamma^{-1}}^{2}\left(T_{1}\right)+L_{\gamma^{-1}}^{1}\left(T_{1}\right) \rightarrow(Y)^{*}
$$

The following result is a quite standard energy-type estimate.

Lemma 4. Let $u$ be a limit of functions that all have compact support. Suppose $u$ solves

$$
H u=f \text { in } S_{1} \text {, }
$$

and that

$$
\begin{gathered}
\iint_{T_{1}} \frac{u^{2}}{-t}+|\nabla u|^{2} \mathrm{~d} \gamma \leq C, \\
\|f\|_{L^{\infty}\left(S_{1}\right)} \leq C
\end{gathered}
$$

Then

$$
\int_{\mathbb{R}^{n}} u^{2}\left(x, t_{0}\right) \mathrm{d} \gamma^{t_{0}} \leq C^{\prime}
$$

for any $t_{0} \in(-4,0)$.

Proof. We first assume that $u$ has compact support. Take $\eta(t) \in C_{0}^{\infty}(\mathbb{R})$ such that $\eta=1$ for $t \in(-1,-2)$ and $\eta(-4)=0$.

Multiplying (3.1) with u $\eta$ and integrating implies for $t_{0} \in(-1,-2)$ :

$$
\int_{-4}^{t_{0}} \int_{\mathbb{R}^{n}}\left(u \eta \Delta u-u_{t} u \eta\right) \mathrm{d} \gamma=\int_{-4}^{t_{0}} \int_{\mathbb{R}^{n}} f u \eta \mathrm{d} \gamma .
$$

Integration by parts implies

$$
\begin{aligned}
& \int_{-4}^{t_{0}} \int_{\mathbb{R}^{n}}\left(-|\nabla u|^{2} \eta-\frac{\left(u^{2} \eta\right)_{t}}{2}+\frac{u^{2} \eta_{t}}{2}\right) \mathrm{d} \gamma \\
& =\int_{-4}^{t_{0}} \int_{\mathbb{R}^{n}} f u \eta \mathrm{d} \gamma .
\end{aligned}
$$


Rearranging the terms, and using the properties of $\eta$ we finally obtain

$$
\int_{\mathbb{R}^{n}}\left(u^{2} \eta\right)\left(x, t_{0}\right) \mathrm{d} \gamma^{t_{0}}=\int_{-4}^{t_{0}} \int_{\mathbb{R}^{n}}\left(-2|\nabla u|^{2} \eta+u^{2} \eta_{t}-2 f u \eta\right) \mathrm{d} \gamma \leq C .
$$

Now let $t_{0} \in(-2,-4)$. Multiplying the equation with $u$ and integrating over $\mathbb{R}^{n} \times\left(t_{0},-1\right)$ yields

$$
\int_{\mathbb{R}^{n}} u^{2}\left(x, t_{0}\right) \mathrm{d} \gamma^{t_{0}}-\int_{\mathbb{R}^{n}} u^{2}(x,-1) \mathrm{d} \gamma^{-1} \leq \int_{t_{0}}^{-1} \int_{\mathbb{R}^{n}} f u \eta \mathrm{d} \gamma \leq C .
$$

We can do the same computations without a function $\eta$, which yields for $t_{0} \in(0,-4)$

$$
\int_{\mathbb{R}^{n}}\left(u^{2}\left(x, t_{0}\right)-u^{2}(x,-4)\right) \mathrm{d} \gamma^{t_{0}}=2 \int_{-4}^{t_{0}} \int_{\mathbb{R}^{n}}\left(-|\nabla u|^{2}-f u\right) \mathrm{d} \gamma \leq C \int_{S_{1}}|u| \mathrm{d} \gamma .
$$

To estimate this, we use that

$$
H\left(|u|-\|f\|_{L^{\infty}\left(S_{1}\right)} t\right) \geq 0
$$

which implies for $C=\|f\|_{L^{\infty}\left(S_{1}\right)}$

$$
\frac{\mathrm{d}}{\mathrm{d} t} \int_{\mathbb{R}^{n}}(|u(x, t)|-C t) \mathrm{d} \gamma^{t} \leq 0,
$$

so that

$$
\int_{\mathbb{R}^{n}}(|u(x, t)|-C t) \mathrm{d} \gamma^{t} \leq \int_{\mathbb{R}^{n}}(|u(x, s)|-C s) \mathrm{d} \gamma^{s},
$$

whenever $t \geq s$. As a consequence

$$
\int_{S_{1}}|u| \mathrm{d} \gamma \leq C+4 \int_{\mathbb{R}^{n}} u(x,-4) \mathrm{d} \gamma^{-4} \leq C+4 \int_{\mathbb{R}^{n}} u^{2}(x,-4) \mathrm{d} \gamma^{-4} \leq C .
$$

Inserted into (3.4) this implies

$$
\int_{\mathbb{R}^{n}} u^{2}\left(x, t_{0}\right) \mathrm{d} \gamma^{t_{0}} \leq C
$$

To finish the proof, take $u$ to be a limit of functions $u_{j}$ with compact support satisfying the hypotheses of the Lemma. Then Fatou's lemma implies that for any $t_{0} \in(-4,0)$

$$
\int_{\mathbb{R}^{n}} u^{2}\left(x, t_{0}\right) \mathrm{d} \gamma^{t_{0}} \leq \liminf _{j \rightarrow \infty} \int_{\mathbb{R}^{n}} u_{j}^{2}\left(x, t_{0}\right) \mathrm{d} \gamma^{t_{0}} \leq C .
$$

In the following lemma we obtain a parabolic counterpart to Almgren's frequency lemma.

Lemma 5. Let $u \in H_{\gamma}^{1}\left(S_{1}\right)$ be caloric in $S_{1}$ and

$$
N(r)=N(r, u)=\frac{\int_{T_{r}}|\nabla u|^{2} \mathrm{~d} \gamma}{\int_{T_{r}} \frac{u^{2}}{-t} \mathrm{~d} \gamma} .
$$

Then $N$ is nondecreasing for $r \in(0,1)$. Furthermore, $N$ is constant if and only if $u$ is parabolic homogeneous.

Proof. The proof is quite standard, and the two main ingredients are partial integration and the Cauchy-Schwarz inequality.

Define $N(r)=A(r) / B(r)$, put $v(x, t)=u\left(r x, r^{2} t\right)$ and

$$
v^{\prime}(x, t)=\frac{d}{d r} v(x, t)=\frac{x}{r} \nabla v(x, t)+\frac{2 t}{r} v_{t}(x, t) .
$$


Moreover, let

$$
A_{R}(r)=\int_{T_{1}}|\nabla v|^{2} \eta \mathrm{d} \gamma, B_{R}(r)=\int_{T_{1}} \frac{v^{2}}{-t} \eta \mathrm{d} \gamma
$$

and also

$$
N_{R}(r)=\frac{A_{R}(r)}{B_{R}(r)}
$$

where $\eta \in C_{0}^{\infty}\left(B_{2 R}\right)$ with $\eta=1$ in $B_{R}$ and $0 \leq \eta \leq 1$. Now we compute, using partial integration and the fact that $\partial_{t} G(x,-t)+\Delta G(x,-t)=0$

and

$$
\begin{aligned}
& A_{R}(r)=\frac{r}{2} \int_{T_{1}} \frac{v v^{\prime}}{-t} \eta \mathrm{d} \gamma+\int_{T_{1}}-v \nabla v \nabla \eta \mathrm{d} \gamma, \\
& A_{R}^{\prime}(r)=r \int_{T_{1}} \frac{\left(v^{\prime}\right)^{2}}{-t} \eta \mathrm{d} \gamma-2 \int_{T_{1}} v^{\prime} \nabla v \nabla \eta \mathrm{d} \gamma,
\end{aligned}
$$

$$
B_{R}^{\prime}(r)=2 \int_{T_{1}} \frac{v v^{\prime}}{-t} \eta \mathrm{d} \gamma
$$

Using the expression for $A_{R}^{\prime}(r)$ we obtain for any $\varepsilon>0$ and any $\alpha>\varepsilon$

$$
\begin{aligned}
A(\beta)-A(\alpha) & =\lim _{R \rightarrow \infty} A_{R}(\beta)-A_{R}(\alpha) \\
& =\lim _{R \rightarrow \infty} \int_{\alpha}^{\beta} r \int_{T_{1}} \frac{\left(v^{\prime}\right)^{2}}{-t} \eta \mathrm{d} \gamma-2 \int_{T_{1}} v^{\prime} \nabla v \nabla \eta \mathrm{d} \gamma \mathrm{d} r \\
& \geq \lim _{R \rightarrow \infty} \int_{\alpha}^{\beta}\left((r-\varepsilon) \int_{T_{1}} \frac{\left(v^{\prime}\right)^{2}}{-t} \eta \mathrm{d} \gamma-\frac{1}{\varepsilon} \int_{T_{1}}|\nabla v|^{2}(-t) \frac{|\nabla \eta|^{2}}{\eta} \mathrm{d} \gamma\right) \mathrm{d} r \\
& \geq \int_{\alpha}^{\beta}\left((r-\varepsilon) \int_{T_{1}} \frac{\left(v^{\prime}\right)^{2}}{-t} \mathrm{~d} \gamma\right) \mathrm{d} r
\end{aligned}
$$

where we used Young's inequality and the fact that $\nabla v \in L_{\gamma}^{2}$, and Fatou's lemma. Hence, letting $\varepsilon \rightarrow 0$ we obtain

$$
A^{\prime}(r) \geq r \int_{T_{1}} \frac{\left(v^{\prime}\right)^{2}}{-t} \mathrm{~d} \gamma
$$

which shows that

is integrable. Therefore,

$$
r \int_{T_{1}} \frac{\left(v^{\prime}\right)^{2}}{-t} \mathrm{~d} \gamma
$$

$$
\begin{array}{r}
N_{R}(s)-N_{R}(t)=\int_{s}^{t} \frac{1}{\left(B_{R}(r)\right)^{2}}\left(A_{R}^{\prime}(r) B_{R}(r)-B_{R}^{\prime}(r) A_{R}(r)\right) \\
=\int_{s}^{t} \frac{r}{\left(B_{R}(r)\right)^{2}}\left(\int_{T_{1}} \frac{\left(v^{\prime}\right)^{2}}{-t} \eta \mathrm{d} \gamma \int_{T_{1}} \frac{v^{2}}{-t} \eta \mathrm{d} \gamma-\left(\int_{T_{1}} \frac{v v^{\prime}}{-t} \eta \mathrm{d} \gamma\right)^{2}\right) \\
+\int_{s}^{t} \frac{r}{\left(B_{R}(r)\right)^{2}}\left(-2 \int_{T_{1}} v^{\prime} \nabla v \nabla \eta \mathrm{d} \gamma B_{R}(r)+\int_{T_{1}}-v \nabla v \nabla \eta \mathrm{d} \gamma B_{R}^{\prime}(r)\right) \\
=\int_{s}^{t} \frac{r}{\left(B_{R}(r)\right)^{2}}\left(\int_{T_{1}} \frac{\left(v^{\prime}\right)^{2}}{-t} \mathrm{~d} \gamma \int_{T_{1}} \frac{v^{2}}{-t} \mathrm{~d} \gamma-\left(\int_{T_{1}} \frac{v v^{\prime}}{-t} \mathrm{~d} \gamma\right)^{2}\right) \\
+\int_{s}^{t} \frac{r}{\left(B_{R}(r)\right)^{2}}\left(-2 \int_{T_{1}} v^{\prime} \nabla v \nabla \eta \mathrm{d} \gamma B_{R}(r)+\int_{T_{1}}-v \nabla v \nabla \eta \mathrm{d} \gamma B_{R}^{\prime}(r)\right),
\end{array}
$$

In addition, the last two terms integrable, due to the estimates

$$
\int_{s}^{t} \frac{r}{B_{R}(r)} \int_{T_{1}}\left|v^{\prime}\right||\nabla v||\nabla \eta| \mathrm{d} \gamma \mathrm{d} r \leq \int_{s}^{t} \frac{r \|\left.\nabla v\right|_{L_{\gamma}^{2}}}{B_{R}(r)} \int_{T_{1}}\left|v^{\prime}\right|^{2} \mathrm{~d} \gamma \mathrm{d} r \leq C
$$


and

$$
\int_{s}^{t} \frac{r}{B_{R}^{2}(r)} \int_{T_{1}}|v \| \nabla v| \mathrm{d} \gamma B_{R}^{\prime}(r) \mathrm{d} r \leq \int_{s}^{t} \frac{r\|v\|_{L_{\gamma}^{2}}^{2}\|\nabla v\|_{L_{\gamma}^{2}}}{B_{R}^{2}(r)} \int_{T_{1}}\left(v^{\prime}\right)^{2} \mathrm{~d} \gamma \mathrm{d} r \leq C .
$$

Also due to (3.6), the first two terms converge. Therefore we obtain

$$
\begin{aligned}
N(s)-N(t) & =\lim _{R \rightarrow \infty} N_{R}(s)-N_{R}(t) \\
& =\int_{s}^{t} \frac{r}{\left(B_{R}(r)\right)^{2}}\left(\int_{T_{1}} \frac{\left(v^{\prime}\right)^{2}}{-t} \mathrm{~d} \gamma \int_{T_{1}} \frac{v^{2}}{-t} \mathrm{~d} \gamma-\left(\int_{T_{1}} \frac{v v^{\prime}}{-t} \mathrm{~d} \gamma\right)^{2}\right) \geq 0,
\end{aligned}
$$

whenever $s \geq t$, due to the Cauchy-Schwarz intequality.

Now suppose that $N\left(r_{1}\right)=N\left(r_{2}\right)$ for $r_{2}>r_{1}$. For $N$ to be constant we need equality in the Cauchy-Schwartz inequality and therefore we have $C(r) v=v^{\prime}$ for $r \in\left(r_{1}, r_{2}\right)$. This implies by the formula for $A$

$$
N(r)=\frac{r C(r)}{2}=N\left(r_{1}\right) .
$$

To see that this implies the correct homogeneity let

$$
u_{r}(x, t)=\frac{u\left(r x, r^{2} t\right)}{r^{\alpha}} .
$$

Then $u$ is parabolic homogeneous of degree $\alpha$ if and only if

$$
\left.\frac{\mathrm{d} u_{r}}{\mathrm{~d} r}=\frac{1}{r^{\alpha}}\left(y \nabla u\left(r x, r^{2} t\right)+2 r t u_{t}\left(r x, r^{2} t\right)-\alpha u\left(r x, r^{2} t\right) / r\right)\right)=0 .
$$

This is equivalent to $\alpha v=r v^{\prime}$ which then implies $2 N\left(r_{1}\right)=\alpha$. Therefore $N$ is constant if and only if $u$ is parabolic homogeneous, with $\alpha=2 N\left(r_{1}\right)$.

In Lemma 4.1 in [Wei01] Weiss presented a monotonicity formula for harmonic functions. Here we need the corresponding caloric result in a special case. Nevertheless we present it in a more general form since it might be of general interest.

Lemma 6. Let $\alpha=1,2,3, \ldots$ and $w \in L_{\gamma}^{2}\left(S_{1}\right)$ be a caloric function in $S_{1}$, which is the limit of functions all having bounded heat operator and compact support. Assume further that $w(0,0)=0, D_{x}^{j} w(0,0)$ vanishes for $0 \leq j \leq \alpha-1$ and $\partial_{t}^{i} w(0,0)$ vanishes for $i=1, \ldots,\lfloor(\alpha-1) / 2\rfloor$. Then

$$
\int_{T_{r}}|\nabla w|^{2} \mathrm{~d} \gamma \geq \frac{\alpha}{2} \int_{T_{r}} \frac{w^{2}}{-t} \mathrm{~d} \gamma
$$

for $0<r<1$ and with equality if and only if $w$ is parabolic homogeneous of degree $\alpha$.

Proof. Assume the contrary, then there exists an $r \in(0,1]$ such that $2 N(r, w)<\alpha$. Since $N(r)$ is nondecreasing $N(r)<\alpha / 2$ as $r \rightarrow 0$. Let

$$
w_{r}=\frac{w\left(r x, r^{2} t\right)}{\left\|\frac{w}{(-t)^{\frac{1}{2}}}\right\|_{L_{\gamma}^{2}\left(T_{r}\right)} .}
$$

We have that $N\left(r_{m}\right)$ is bounded for some sequence $r_{m} \rightarrow 0$. Therefore,

$$
\begin{gathered}
\left\|\frac{w_{r_{m}}}{(-t)^{\frac{1}{2}}}\right\|_{L_{\gamma}^{2}\left(T_{1}\right)}=1, \\
\left\|\nabla w_{r_{m}}\right\|_{L_{\gamma}^{2}\left(T_{r}\right)} \leq\left\|\nabla w_{r_{m}}\right\|_{L_{\gamma}^{2}\left(T_{1}\right)} \leq C \text { for } r<1 .
\end{gathered}
$$


Lemma 4 together with interior estimates now imply that we have local uniform boundedness in $C^{\infty}\left(\mathbb{R}^{n} \times(-4,0]\right)$. Applying Lemma 4 again and using the fact that $\partial_{t} w_{r_{m}}=\Delta w_{r_{m}} \in \nabla L_{\gamma}^{2}$, we have

$$
\begin{aligned}
\|w\|_{L^{\infty}\left((-4,-1), L_{\gamma^{t}}^{2}\left(\mathbb{R}^{n}\right)\right)} & \leq C, \\
\left\|\partial_{t} w_{r_{m}}\right\|_{L^{1}\left((-4,-1), Y^{*}\right)} & \leq C,
\end{aligned}
$$

Now define $\hat{w_{m}}(x, t)=w_{r_{m}}(x \sqrt{-t}, t)$. Then

$$
\begin{aligned}
\nabla \hat{w}_{m}(x, t) & =\sqrt{-t} \nabla w_{r_{m}}(x \sqrt{-t}, t) \\
\partial_{t} \hat{w}_{m}(x, t) & =\partial_{t} w_{r_{m}}(x \sqrt{-t}, t)+\frac{x}{2 \sqrt{-t}} \nabla w_{r_{m}}(x \sqrt{-t}, t) \\
& \subset \nabla L_{\gamma^{-1}}^{2}\left(T_{1}\right)+L_{\gamma^{-1}}^{1}\left(T_{1}\right) \subset L^{1}\left((-4,-1), Y^{*}\right) .
\end{aligned}
$$

This implies that have the uniform estimates

$$
\left\|\frac{\hat{w}_{m}}{(-t)^{\frac{1}{2}}}\right\|_{L_{\gamma^{-1}}^{2}}+\left\|\nabla \hat{w}_{m}\right\|_{L_{\gamma^{-1}}^{2}\left(T_{1}\right)}+\left\|\partial_{t} \hat{w}_{m}\right\|_{L^{1}\left((-4,-1), Y^{*}\right)} \leq C .
$$

For $X_{0}=H_{\gamma^{s}}^{1}\left(\mathbb{R}^{n}\right), X=L_{\gamma^{s}}^{2}\left(\mathbb{R}^{n}\right)$ and $X_{1}=Y^{*}$ we can use Lemma 3 . This implies that for a subsequence $\hat{w}_{m}$ converges in $L_{\gamma}^{2}\left(T_{1}\right)$, and so does $w_{r_{m}}$. Therefore

$$
w_{0}=\lim _{m \rightarrow \infty} w_{r_{m}}
$$

is a caloric function in $S_{1}$, with

$$
\begin{aligned}
& D_{x}^{j} w_{0}(0,0)=0 \text { for } j=0,1,2, \ldots, \alpha-1, \\
& \partial_{t}^{i} w_{0}(0,0)=0 \text { for } i=1, \ldots,\lfloor(\alpha-1) / 2\rfloor
\end{aligned}
$$

and

$$
\left\|\frac{w_{0}}{(-t)^{\frac{1}{2}}}\right\|_{L_{\gamma}^{2}\left(T_{1}\right)}=1
$$

Furthermore,

$$
N\left(\beta, w_{r_{m}}\right)-N\left(\alpha, w_{r_{m}}\right)=N\left(\beta r_{m}, w\right)-N\left(\alpha r_{m}, w\right) \rightarrow 0
$$

Therefore,

$$
N(r)\left(w_{0}\right)=N\left(0^{+}\right) \text {for } 0<r<1,
$$

which implies that, by Lemma $5, w_{0}$ must be parabolic homogeneous of degree $2 N(0+) \in[0, \alpha)$. From the regularity at the origin we have that $2 N\left(0^{+}\right) \in \mathbb{N}$. Since $2 N(0+)<\alpha$ and

$$
\begin{gathered}
D_{x_{j}} w_{0}(0,0)=0 \text { for } j=0,1, \ldots, \alpha-1, \\
\partial_{t}^{i} w(0,0)=0 \text { for } i=1, \ldots,\lfloor(\alpha-1) / 2\rfloor,
\end{gathered}
$$

this implies that $w_{0}=0$, which is a contradiction.

Hence, $2 N(r) \geq \alpha$ for $0<r \leq 1$ and $2 N(s)=\alpha$ implies that $N$ is constant in $0 \leq r \leq s$ and therefore $w$ is parabolic homogeneous of degree $\alpha$ in $0 \leq r \leq s$.

\section{The optimal REgularity}

In this section we prove the main theorem. First we obtain some estimates which we will need. The lemma below proves that under the assumptions in Theorem 1, we have quadratic growth at the level $r_{0}$. 
Lemma 7. Let $u \in P_{4}\left(M_{1}, M_{2}\right), \psi \in C_{0}^{\infty}\left(B_{\frac{3}{4}}\right)$ such that $\psi=1$ on $B_{\frac{1}{2}}$ and $v=u \psi$. Given $\varepsilon>0$, there exists $\lambda_{\varepsilon, M_{i}}>0$ and $C_{\varepsilon, M_{i}}<\infty$ such that if for some $0<r_{0}<\lambda_{\varepsilon, M_{i}}$

$$
\delta\left(r_{0} / 2, v\right)>\varepsilon \text { and } W\left(r_{0} ; v, f\right) \leq 2 A_{n}-\varepsilon
$$

then

$$
\int_{T_{r_{0}}} \frac{v^{2}}{-t} \mathrm{~d} \gamma \leq C_{\varepsilon, M_{i}} r_{0}^{4}
$$

Proof. Suppose towards a contradiction that there exists a sequence $r_{m} \rightarrow 0$ and a sequence of functions $u_{m} \in P_{1}\left(M_{1}, M_{2}\right)$ with $v_{m}=\psi u_{m}$, such that

$$
\begin{array}{r}
\delta\left(r_{m} / 2, v_{m}\right)>\varepsilon, \\
W\left(r_{m} ; v_{m}, f\right) \leq W_{0}, \\
C_{m}=\frac{\left\|\frac{v_{m}}{(-t)^{\frac{1}{2}}}\right\|_{L_{\gamma}^{2}\left(T_{r_{m}}\right)}}{r_{m}^{2}} \rightarrow \infty .
\end{array}
$$

Now we use the following rescaling

$$
w_{r_{m}}(x, t)=\frac{v_{m}\left(r_{m} x, r_{m}^{2} t\right)}{C_{m} r_{m}^{2}} .
$$

Then $w_{r_{m}}$ is a solution to (1.1) with

$$
f_{m}^{\prime}=\frac{f_{u_{m}}\left(r_{m} x, r_{m}^{2} t\right)}{C_{m}} \rightarrow 0 \text { in } B_{1 / 2 r^{m}} \times\left(-4 / r_{m}^{2}, 0\right] .
$$

We also have

$$
\begin{aligned}
\delta\left(1 / 2, w_{r_{m}}\right) & \geq \varepsilon, \\
\left\|\frac{w_{r_{m}}}{(-t)^{\frac{1}{2}}}\right\|_{L_{\gamma}^{2}\left(T_{1}\right)} & =1 .
\end{aligned}
$$

Moreover, the energy assumption implies

$$
W\left(1 ; w_{r_{m}}, f_{m}^{\prime}\right)=\frac{W\left(r_{m} ; w_{r_{m}}, f_{u_{m}}\right)}{C_{m}^{2}} \leq \frac{W_{0}}{C_{m}^{2}},
$$

and therefore, in particular

$$
\int_{T_{1}}\left|\nabla w_{r_{m}}\right|^{2} \mathrm{~d} \gamma \leq \int_{T_{1}}\left(\frac{w_{r_{m}}^{2}}{t}+\frac{2 A_{n}-\varepsilon}{C_{m}^{2}}+\frac{1}{C_{m}} \sup _{T_{r_{m}}} f_{u_{m}}\right) \mathrm{d} \gamma
$$

where the last two terms vanish in the limit, and the right hand side is bounded. We observe that since $u$ is locally in $C_{x}^{1, \alpha} \cap C_{t}^{\alpha}\left(Q_{2}^{-}\right)$(due to local estimates for $f$ bounded, see Theorem 4.8 in [Lie96]), $H v \in L^{\infty}\left(S_{1}\right)$. Therefore, $H v_{m} \in L^{\infty}\left(S_{1}\right)$. In what follows we argue as in Lemma 6 . We have

$$
\begin{aligned}
& \left\|\frac{w_{r_{m}}}{(-t)^{\frac{1}{2}}}\right\|_{L_{\gamma}^{2}\left(T_{1}\right)}=1, \\
& \left\|\nabla w_{r_{m}}\right\|_{L_{\gamma}^{2}\left(T_{1}\right)} \leq C .
\end{aligned}
$$

Lemma 4 together with interior estimates implies that $w_{r_{m}}$ is locally uniformly bounded in $C_{t}^{1, \alpha} \cap C_{t}^{0, \alpha}\left(\mathbb{R}^{n} \times(-4,0]\right)$. In addition, Lemma 4 implies

$$
\left\|w_{r_{m}}\right\|_{L^{\infty}\left((-4,0), L_{\gamma^{t}}^{2}\left(\mathbb{R}^{n}\right)\right)} \leq C .
$$

The equation for $v_{m}$ gives

$$
\partial_{t} v_{m}=\Delta v_{m}-H v_{m} \subset \nabla L_{\gamma}^{2}\left(T_{1}\right)+L^{\infty}\left(T_{1}\right) .
$$


Applying Lemma 3 as in the proof of Lemma 6, we find a subsequence converging in $L_{\gamma}^{2}\left(T_{1}\right)$. Thus

$$
\int_{T_{1}} \frac{w_{0}^{2}}{-t} \mathrm{~d} \gamma=1
$$

where

$$
w_{0}=\lim _{m \rightarrow \infty} w_{r_{m}} .
$$

If we pass (4.2) to the limit we obtain by the weak convergence

$$
\int_{T_{1}}\left|\nabla w_{0}\right|^{2} \mathrm{~d} \gamma \leq \int_{T_{1}} \frac{w_{0}^{2}}{-t} \mathrm{~d} \gamma .
$$

We also have that (4.1) implies that $w_{0}$ is caloric in $S_{1}$ and that both $w_{0}$ and $\left|\nabla w_{0}\right|$ are zero at the origin. This together with Lemma 6 implies

$$
\int_{T_{1}}\left|\nabla w_{0}\right|^{2} \mathrm{~d} \gamma \geq \int_{T_{1}} \frac{w_{0}^{2}}{-t} \mathrm{~d} \gamma
$$

Therefore, again by Lemma $6,(4.4)$ and (4.5) are possible if and only if $w_{0}$ is a parabolic homogeneous quadratic polynomial in $T_{1}$, and thus also in $Q_{1}^{-}$by unique continuation. Moreover, the local uniform convergence gives

$$
\delta\left(1 / 2, w_{0}\right) \geq \varepsilon .
$$

But we now that $w_{0}$ can (up to tilting the coordinates) be written as

$$
w_{0}=\sum_{1}^{n} \lambda_{i} x_{i}^{2}+\alpha t .
$$

So unless $\alpha=\lambda_{i}=0$ for all $i$, then the set $\{u=|\nabla u|=0\}$ contains only the origin. Therefore $w_{0}$ has to be identically zero, which contradicts (4.3).

The next result is very similar to Lemma 8, but here we obtain a fix constant in the quadratic growth, and also an estimate of how close the solution is to a halfspace solution.

Lemma 8. Let $u \in P_{4}\left(M_{1}, M_{2}\right), \psi \in C_{0}^{\infty}\left(B_{\frac{3}{4}}\right)$ such that $\psi=1$ on $B_{\frac{1}{2}}$ and $v=u \psi$. Given $\varepsilon>0, d>0$ and $\beta_{0}>0$, there exists $\lambda=\lambda_{\varepsilon, M_{i}, d, \beta_{0}}>0$ such that if for some $0<r_{0}<\lambda$

$$
\delta\left(r_{0} / 2, v\right)>\varepsilon \text { and } W\left(r_{0} ; v, f\right) \leq 2 A_{n}-\varepsilon
$$

then

$$
\sup _{Q_{r_{0}}^{-}}|v| \leq r_{0}^{2}
$$

and

$$
\|v-h\|_{L^{\infty}\left(Q_{r_{0} d}^{-}\right)}<\beta_{0}\left(r_{0} d\right)^{2} .
$$

Here $h$ is again a half-space solution, with $f=1$.

Proof. As usual, we argue by contradiction. If the assertion does not hold, then there is a sequence $r_{j} \rightarrow 0$ such that the hypothesis hold for $r_{j}$ but still either

$$
\sup _{Q_{r_{j}}^{-}}|v|>r_{j}^{2}
$$

or

$$
\|v-h\|_{L^{\infty}\left(Q_{r_{j} d}^{-}\right)}>\beta_{0} r_{j}^{2} d^{2},
$$


for all halfspace solutions $h$. Define the rescaled functions

$$
w_{j}(r, x)=w_{r_{j}}(x, t)=\frac{u\left(r_{j} x, r_{j}^{2} t\right)}{r_{j}^{2}}
$$

as usual. Then, since $H v_{j} \in L^{\infty}\left(\mathbb{R}^{n} \times \mathbb{R}^{+}\right.$) (due to the local estimates on $u$ ), we also have $H w_{j} \in L^{\infty}\left(\mathbb{R}^{n} \times \mathbb{R}^{+}\right)$uniformly. Moreover,

$$
\begin{gathered}
\sup _{Q_{1}^{-}}\left|w_{j}\right|>1, \\
\left\|w_{j}-h\right\|_{L^{\infty}\left(Q_{d}^{-}\right)}>\beta_{0} d^{2},
\end{gathered}
$$

for all halfspace solutions $h$. By Lemma 7

$$
\int_{T_{1}} \frac{w_{j}^{2}}{-t} \mathrm{~d} \gamma \leq C_{\varepsilon, M_{i}}
$$

In addition, we have

$$
H w_{j}=f\left(r_{j} x, r_{j}^{2} t\right) \chi_{\Omega_{j}} \text { in } B \frac{1}{2 r_{j}} \times\left(-4 / r_{j}, 0\right),
$$

and from the assumption on the Weiss energy

$$
\int_{T_{1}}\left|\nabla w_{j}\right|^{2} \mathrm{~d} \gamma \leq C
$$

Now we proceed as in the proof Lemma 6; Lemma 4 implies

$$
\int_{\mathbb{R}^{n}} w_{j}^{2}(x, t) \mathrm{d} \gamma^{t} \leq C
$$

for all $t \in(-4,0)$. Using the equation for $w_{j}$ we have

$$
\partial_{t} w_{j}=-H w_{j}+\Delta w_{j} \subset L^{\infty}\left(T_{1}\right)+\nabla L_{\gamma}^{2}\left(T_{1}\right) .
$$

Therefore, by Lemma 3 applied as in Lemma 6, and by interior estimates (Theorem 6.17 combined with Theorem 4.8 in [Lie96]), up to a subsequence, $w_{j}$ converges in $L_{\gamma}^{2}\left(T_{1}\right)$ and locally in $C_{x}^{1, \alpha} \cap C_{t}^{\alpha}\left(\mathbb{R}^{n} \times(-4,0]\right)$. The limit function $w_{0}$ satisfies

$$
\sup _{Q_{1}^{-}}\left|w_{0}\right| \geq 1
$$

or

$$
\left\|w_{0}-h\right\|_{L^{\infty}\left(Q_{d}^{-}\right)} \geq \beta_{0} d^{2},
$$

and

$$
H w_{0}=f(0,0) \chi_{\Omega}=\chi_{\Omega} \text { in } \mathbb{R}^{n} \times(-4,0) .
$$

Moreover, due to the almost monotonicity of the Weiss' functional

$$
W\left(s ; w_{0}, 1\right)=\lim _{j \rightarrow \infty} W\left(s r_{j} ; v, f\right)=W(0 ; v, f)<2 A_{n}-\varepsilon,
$$

for all $s>0$. Hence, $w_{0}$ is parabolic homogeneous of degree 2. This implies, by the classification of global solutions (Theorem I [CPS04]) that $w_{0}=h$. This is a contradiction to both (4.6) and (4.7).

Remark 2. We remark that Lemma 7 and Lemma 8 will still be valid, if we have a solution with compact support and defined everywhere (so that the Weiss energy is well-defined), and if we assume

$$
W(r ; v, f) \leq 2 A_{n}-\varepsilon
$$

for all $r \leq r_{0}$, and also that $W(r, v, f)$ has a limit as $r \rightarrow 0$.

In the following lemma we prove that if a solution is sufficiently close to a halfspace solution, then the set $\Lambda(u)$ cannot be too small. 
Lemma 9. Fix $d>0$ and let $u \in P_{1}\left(M_{1}, M_{2}\right)$. Suppose

$$
\|u-h\|_{L^{\infty}\left(Q_{1}^{-}\right)} \leq \beta_{0},
$$

where $h$ is a halfspace solution. Then, if $\beta_{0}$ is sufficient small (depending on $M_{2}$ and d), we will have

$$
\delta(u, 1 / 2)>1 / 2
$$

Proof. Suppose the contrary is true. Then there are halfspace solutions $h_{m}$ and a subsequence of functions $u_{m} \in P_{1}\left(M_{1_{m}}, M_{2}\right)$ such that

$$
\lim _{m \rightarrow \infty}\left\|u_{m}-h_{m}\right\|_{L^{\infty}\left(Q_{1}^{-}\right)}=0
$$

and

$$
\delta\left(u_{m}, 1 / 2\right) \leq 1 / 2 .
$$

If $h_{m}$ converges to $h_{0}$ it follows by interior estimates (Theorem 4.8 in [Lie96]) that there is a subsequence $u_{m}$, that converges to $h_{0}$ in $C_{x, \mathrm{loc}}^{1, \alpha} \cap C_{t, \text { loc }}^{0, \alpha}\left(Q_{1}^{-}\right)$. For this sequence we have

$$
\lim _{m \rightarrow \infty} \delta\left(u_{m}, 1 / 2\right)=\delta\left(h_{0}, 1 / 2\right)=1
$$

which is a contradiction.

We are now ready to give the proof of our main theorem. The idea of the proof is very similar to the one of the proof of Theorem A1 in [PS07a].

Proof of Theorem 1. To make the proof more clear we divide into several steps. In what follows we will take $d=1 / 8$.

Step 1: Bound on $W$. First we establish an upper bound for $W(r ; v, f)$. To prove the estimate, take $u \in P_{1}\left(M_{1}, M_{2}\right), \psi \in C_{0}^{\infty}\left(B_{1}\right), \psi=1$ in $B_{\frac{1}{2}}$ and let $v=u \psi$. Since in the monotonicity formula (Proposition 1) $F(r)$ is a continuous function that vanishes at the origin, there exists a positive constant $r_{\varepsilon, M_{1}, M_{2}}$ such that

$$
W\left(r_{1} ; v, f\right) \leq W\left(r_{2} ; v, f\right)+\varepsilon / 2,
$$

for every $0<r_{1} \leq r_{2} \leq r_{\varepsilon, M_{1}, M_{2}}$. Therefore it follows that if $W\left(r_{0} ; v, f\right) \leq 2 A_{n}-\varepsilon$ for some $0<r_{0}<r_{\varepsilon, M_{1}, M_{2}}$ then

$$
W(r ; v, f) \leq 2 A_{n}-\varepsilon / 2,
$$

for every $0<r \leq r_{0}$.

Step 2: First bound on $\delta$. The second step of the proof is to find a lower bound of the thickness function of a rescaled version of $v$.

As in Lemma 8 take $\varepsilon>0$ and $\beta_{0}>0$ small enough (as in Lemma 9 ). Then to this end chose

$$
\lambda=\min \left(\lambda_{\varepsilon, M_{1} / d^{2 k}, M_{2}, \beta_{0}}, \lambda_{\varepsilon, 1 / d^{2 k}, M_{2}, \beta_{0}}\right),
$$

where $k$ is an integer such that

$$
d^{k} \leq r_{0} \leq d^{k-1}
$$

Then if for some $0<r_{0}<\lambda$

$$
\delta\left(r_{0} / 2, v\right)>\varepsilon \text { and } W\left(r_{0} ; v, f\right) \leq 2 A_{n}-\varepsilon / 2
$$

we have by Lemma 8

$$
\sup _{Q_{r_{0}}^{-}}|v| \leq r_{0}^{2},
$$

and

$$
\|v-h\|_{L^{\infty}\left(Q_{r_{0} d}^{-}\right)} \leq \beta_{0} r^{2} d^{2} .
$$


Then Lemma 9 implies that

$$
\delta\left(r_{0} / 2, v_{d}\right)=\delta\left(\frac{1}{2}, v_{r_{0} d}\right)>\frac{1}{2}
$$

By the simplest possible estimate we have

$$
\sup _{Q_{4 d}^{-}}|v| \leq M_{1}
$$

so $v_{d} \in P_{4}\left(M_{1} / d^{2}, M_{2}\right)$.

Step 3: Repeat Step 1 and Step $2(k-1)$ times. We see that all the assumptions in Lemma 8 (see also the remark after Lemma 8) are again satisfied for $v_{d}$, except that $M_{1}$ is changed to $M_{1} / d^{2}$. So we iterate Step 1 and Step 2 repeatedly to $v_{d}, v_{d^{2}}$ until $v_{d^{k}}$. Then we have all along the way that for $i \leq k$

$$
v_{d^{i}} \in P_{4}\left(M_{1} / d^{2 i}, M_{2}\right)
$$

and

$$
\delta\left(r_{0} / 2, v_{d^{i}}\right)>\frac{1}{2}
$$

Step 4: The $k$-th iteration. Now we wish to estimate the supremum of $v_{d^{k}}$, for which we use the relation between $r_{0}$ and $d$ for as follows

$$
\sup _{Q_{4}^{-}}\left|v_{d^{k}}\right|=\frac{1}{d^{2 k}} \sup _{Q_{4 d^{k}}^{-}}|v| \leq \frac{1}{d^{2 k}} \sup _{Q_{r_{0}}^{-}}|v| \leq \frac{r_{0}^{2}}{d^{2 k}} \leq 64
$$

by (4.8). Hence, $v_{d^{k}} \in P_{4}\left(64, M_{2}\right)$ and also

$$
\delta\left(r_{0} / 2, v_{d^{k}}\right)>\varepsilon
$$

and

$$
W\left(r ; v_{d^{k}}, f\left(d^{k} x, d^{2 k} t\right)\right) \leq 2 A_{n}-\varepsilon
$$

for all $r \leq r_{0}$, as before.

Step 5: Continue the iterations. Now we can iterate Step 1 and Step 2 to $v_{d^{k}}$ $k$ times to obtain

$$
v_{d^{k+i}} \in P_{4}\left(64 / d^{2 i}, M_{2}\right)
$$

and

for $i \leq k$.

$$
\delta\left(r_{0} / 2, v_{d^{k+i}}\right)>\frac{1}{2}
$$

And when we reach $d^{k}$ we use the estimate at level $r_{0}$ of $v_{d^{k}}$ gotten from applying Lemma 8 to $v_{d^{k}}$ which gives us again that

$$
v_{d^{2 k}} \in P_{4}\left(64, M_{2}\right),
$$

so that the constants never blow up, they are between 64 and $64 / d^{2 k}$. Thus for all $j=0,1,2, \ldots$ we obtain

$$
d^{2 j} \sup _{Q_{r_{0}}^{-}}\left|v_{d^{j}}\right|=\sup _{Q_{r_{0} d^{j}}^{-}}|v| \leq\left(r_{0} d^{j}\right)^{2} .
$$

This implies that we have for all $r<r_{0}$ the estimate

$$
\sup _{Q_{r}^{-}}|v| \leq C r^{2}
$$

where $C$ is a fixed constant.

Step 5: Apply for points close the origin. Since $f \in C^{0, \beta}\left(Q_{1}^{-}\right)$, interior estimates applies away from the free boundary. Therefore, we only have to obtain 
regularity close to the free boundary. In order to obtain the estimate, take a free boundary point $\left(x_{0}, t_{0}\right) \in \Gamma \cap Q_{c_{0}}^{-}$and introduce the scaling

$$
w(x, t)=\frac{v\left(x+x_{0}, t+t_{0}\right)}{f\left(x_{0}, t_{0}\right)} \in P_{2}\left(2 M_{1}, 2 M_{2}\right) .
$$

If $c_{0}=C\left(\varepsilon, M_{1}, M_{2}, r_{0}\right)$ is small enough we have the following for $w$ :

$$
\delta\left((3 / 4) r_{0}, w\right) \geq \varepsilon / 2
$$

and

$$
W\left(r_{0} ; w, f\right) \leq \frac{2 A_{n}-\varepsilon / 2}{\left(f\left(x_{0}\right)\right)^{2}} \leq 2 A_{n}-\varepsilon / 4
$$

This is similar to (1.2) and we can adjust the lemmata and Step 1 to Step 4 for these assumptions. Using this result we will find that $v$ satisfies

$$
|v(x, t)| \leq C \operatorname{dist}((x, t), \Gamma)^{2} \text { for }(x, t) \in Q_{c_{0}}^{-} .
$$

To do the final estimates for $v$ take $\left(x_{1}, t_{1}\right) \in Q_{c_{0}}^{-}$, let $\rho=\operatorname{dist}\left(\left(x_{1}, t_{1}\right), \Gamma\right)$ and

$$
v_{\rho}(x, t)=\frac{v\left(\rho x, \rho^{2} t\right)}{\rho^{2}} \text {. }
$$

Then $v_{\rho} \in L^{\infty}\left(Q_{1}^{-}\right)$and

$$
H v=f \text { in } Q_{1}^{-} .
$$

By Schauder estimates (see for instance Theorem 4.9 in [Lie96]) we have

$$
\|v\|_{C_{x}^{1,1} \cap C_{t}^{0,1}\left(Q_{\rho}^{-}\left(x_{1}, t_{1}\right)\right)}=\left\|v_{\rho}\right\|_{C_{x}^{1,1} \cap C_{t}^{0,1}\left(Q_{1}^{-}\right)} \leq C,
$$

which is independent of $\rho$.

\section{ACKNOWLedgements}

The authors would like to thank Henrik Shahgholian for suggesting the problem and also for all the ideas and helpful discussions along the development of this paper. They are also grateful to Régis Monneau for suggesting the use of Simon's lemma in order to obtain compactness.

\section{REFERENCES}

[Bla01] Ivan Blank, Sharp results for the regularity and stability of the free boundary in the obstacle problem, Indiana Univ. Math. J. 50 (2001), no. 3, 1077-1112.

[BS03] Ivan Blank and Henrik Shahgholian, Boundary regularity and compactness for overdetermined problems, Ann. Sc. Norm. Super. Pisa Cl. Sci. (5) 2 (2003), no. 4, 787-802.

[CKS00] Luis A. Caffarelli, Lavi Karp, and Henrik Shahgholian, Regularity of a free boundary with application to the Pompeiu problem, Ann. of Math. (2) 151 (2000), no. 1, 269-292.

[CPS04] Luis Caffarelli, Arshak Petrosyan, and Henrik Shahgholian, Regularity of a free boundary in parabolic potential theory, J. Amer. Math. Soc. 17 (2004), no. 4, 827-869 (electronic).

[Hoo81] James G. Hooton, Compact Sobolev imbeddings on finite measure spaces, J. Math. Anal. Appl. 83 (1981), no. 2, 570-581.

[Lie96] Gary M. Lieberman, Second order parabolic differential equations, World Scientific Publishing Co. Inc., River Edge, NJ, 1996.

[LS09] Peter Laurence and Sandro Salsa, Regularity of the free boundary of an American option on several assets, Comm. Pure Appl. Math. 62 (2009), no. 7, 969-994.

[PS07a] Arshak Petrosyan and Henrik Shahgholian, Geometric and energetic criteria for the free boundary regularity in an obstacle-type problem, Amer. J. Math. 129 (2007), no. 6, $1659-1688$.

[PS07b] _ Parabolic obstacle problems applied to finance, Recent developments in nonlinear partial differential equations, 2007, pp. 117-133.

[Sim87] Jacques Simon, Compact sets in the space $L^{p}(0, T ; B)$, Ann. Mat. Pura Appl. (4) 146 (1987), 65-96. 
[Wei01] G. S. Weiss, An obstacle-problem-like equation with two phases: pointwise regularity of the solution and an estimate of the Hausdorff dimension of the free boundary, Interfaces Free Bound. 3 (2001), no. 2, 121-128.

[Wei99]_, Self-similar blow-up and Hausdorff dimension estimates for a class of parabolic free boundary problems, SIAM J. Math. Anal. 30 (1999), no. 3, 623-644 (electronic). 